\title{
Prevalence of tuberculosis, HIV/AIDS, and hepatitis; in a prison of Balochistan: a cross- sectional survey
}

\author{
Ahmad Wali ${ }^{1,2^{*}}$ (D), Dawood Khan ${ }^{3}$, Nauman Safdar ${ }^{4,5}$, Zeenat Shawani ${ }^{1}$, Razia Fatima ${ }^{2}$, Aashifa Yaqoob², \\ Aurangzeb Qadir ${ }^{2}$, Sultan Ahmed ${ }^{1}$, Hamayun Rashid ${ }^{6}$, Bashir Ahmed ${ }^{1}$ and Shereen Khan ${ }^{7}$
}

\begin{abstract}
Background: Human Immunodeficiency Virus (HIV), Hepatitis, and Tuberculosis (TB) are three primary communicable infections have the likely potential to cause severe morbidity in prison settings. The prison has the most favorable environment for the transmission of infections. We conducted this survey to determine the prevalence and feasibility of rapid diagnostic tests in an active screening of these infectious diseases in prison.

Methods: This cross-sectional survey conducted in central Jail Gaddani, one of the largest prisons in the Balochistan province of Pakistan. All prisoners, jail staffs, and staff's family members participated. Informed consent obtained from each participant before the screening. Van equipped with digital X-ray linked with Computer-Aided Detection for TB (CAD4TB) software used for testing. Sputum samples tested on Xpert for MTB/RIF assay and blood specimens collected for HIV and hepatitis serology. Diagnosed TB patients enrolled for treatment at Basic Management Unit (BMU), reactive on hepatitis Rapid Diagnostic Tools (RDTs) were referred for further testing and management, while HIV reactive referred to Anti Retro Viral (ARV) center for Anti Retro Viral Treatment (ART).

Results: A total of 567 participants offered screening, 63\% (356) prisoners, 23\% (129) staff's family members, and 14\% (82) jail staffs. Among tested 10.3\% (58/562) were hepatitis seropositive (Hepatitis-C 41 [7.29\%] Hepatitis-B, 16 [2.84\%] Hepatitis B\&C both, 01 [0.17\%]). In reactive participants, 49 were prisoners, 08 were jail staffs, and 01 was the staff's family member. HIV seropositive was 4\% (24/566), and all were prisoners. Almost 99\% (565/567) screened by digital X-ray, 172 (30\%) were with abnormal CAD4TB suggestion (score > 50), out of them sputum of 26\% (148) tested on Xpert, and 2\% (03) found Mycobacterium tuberculosis Positive (MTB+). A total of five TB patients were detected; out of two were diagnosed clinically. Co-morbidities observed in 15 patients, (01 TB/HIV co-infected, 12 $\mathrm{HIV} / \mathrm{HCV}, 01 \mathrm{HIV} / \mathrm{HBV}$, and $01 \mathrm{HBV} / \mathrm{HCV}$ ).

Conclusion: The high frequency of infectious diseases in prison is alarming. For limiting the transmission of infections among prison and community, immediate steps are needed to be taken for improvement of prisons condition by application of recommended screening protocols at the time of the first entry of prisoners in prisons.
\end{abstract}

Keywords: Prison, HIV, Hepatitis, Tuberculosis, Screening, Prevalence, CAD4TB, Active-case-finding

\footnotetext{
* Correspondence: dr_wali786@yahoo.com

${ }^{1}$ Provincial Tuberculosis Control Program, Health Department Government of

Balochistan, Building Western Bypass Link Brewery Road, Quetta, Balochistan,

Pakistan

${ }^{2}$ National Tuberculosis Control Program, Ministry of NHSR\&C, Islamabad,

Pakistan

Full list of author information is available at the end of the article
}

(c) The Author(s). 2019 Open Access This article is distributed under the terms of the Creative Commons Attribution 4.0 International License (http://creativecommons.org/licenses/by/4.0/), which permits unrestricted use, distribution, and reproduction in any medium, provided you give appropriate credit to the original author(s) and the source, provide a link to the Creative Commons license, and indicate if changes were made. The Creative Commons Public Domain Dedication waiver (http://creativecommons.org/publicdomain/zero/1.0/) applies to the data made available in this article, unless otherwise stated. 


\section{Background}

Prisons act as a reservoir for infectious diseases and inflating the disease into the community through staffs, visitors, and releases of inadequately treated prisoners [1-3]. According to World Prison Brief, in 2015; more than 10.35 million prisoners either as pre-trial/remand /convicted or sentenced are under in penal institutions in 223 prisons. During the same period; about 80,169 persons (pre-trial/remand/ convicted/sentenced) were in prisons in Pakistan, which was $171 \%$ occupancy level of actual (40705) official capacity of the prison system [4].

.Prisons reported with inadequate medical care services, overcrowding and poor hygienic conditions in general [5]. The injurious/high-risk activities i.e., injecting drugs, alcoholism, unprotected and multiple-partner sexual activity, barbering, and smoking, are associated with overcrowded prisons. Which inversely contribute to the poor health of the individual and higher risk of infection transmission and development of disease in later ages [1, 6-10]. Globally, the majority of the prisoners belong to age group of 15-44 years and of low socioeconomic status and having inadequate education. They are also frequently subjected to avoidable health risks: through lack of access to screening, immunization, and active case-finding programs [10-12]. Screening of prisoners and jails staff and staff's family members could provide a high-yield opportunity for early disease detection and timely treatment to control infection in prisons and the general population [3].

.Pakistan is a double disease burden country; facing the challenge of the common epidemic and endemic infectious diseases [13]. Globally; Pakistan is the 5th highest TB burden and 2nd highest viremic infection country [14, 15]. In 2017; a total 368,897 TB cases notified against the estimated burden of 525,000 [16]. HIV and Acquired Immunodeficiency Deficiency Syndrome (AIDS) epidemic among the general population in Pakistan is less than $0.1 \%$. There is a shift from "Low prevalence in key population' to a more concentrated epidemic in Pakistan [10]. In 2016; an estimated 130,000 (120,000-150,000) people living with HIV in Pakistan [17]. In 2017; more than 10 million people infected by Hepatitis in Pakistan [18]. Hepatitis B\&C prevalence are 2.5 and $4.8 \%$ respectively, with co-infection combined rate of $7.6 \%$ in the general population in Pakistan [15].

.Prisons in Balochistan like other parts of the country are also overcrowded and might be one of the key contributing factors to poor prison conditions and health status $[12,19,20]$. Preventive programs like TB, AIDS, and hepatitis control programs don't provide permanent screening and management setups within the prisons.

Globally in prisons, high prevalence, and wide transmission of HIV, Hepatitis-B Virus (HBV), Hepatitis-C Virus $(\mathrm{HCV})$ and $\mathrm{TB}$ is witnessed than the general population. In addition to the challenges faced in a prison setting, there are opportunities for early detection and management of these highly infectious diseases [4, 10-12, 18, 21, 22]. In south Asia, prisons recognized as hubs of infectious diseases. Statistics on the burden of HIV, HBV, HCV, and TB in prisons have not well documented, and therefore, information comes mainly from studies conducted in developed countries [20, 23]. Research studies in Pakistan have focused on disease detection, treatment, community linkages, health education, and prevention for prison inmates $[3,5,20,22,24]$. Limited shreds of evidence founded for research, survey or screening through active case finding and utilization of advance screening, and diagnostic tools with high sensitivity and specificity for the assessment of the prevalence of three major infectious diseases collectively. This is likely to underestimate the overall magnitude of the problem; because a large number of prisons and the prison population are there.

This study aimed to assess the prevalence of three infectious diseases HIV, Hepatitis B-C, and TB among prisoners, jail staffs, and staff's family members.

\section{Methods}

It was a cross-sectional study. Permission obtained from the Inspector General (IG) Prisons through provincial home department. During camp, informed written consent included questionnaires pursued from each of the participants. Parents in case of children (signature, writing name, and thumb impression) before administration of screening to collect personal and socio-demographic data.

\section{Study site}

This study conducted in central Jail Gaddani, district Lasbela of Balochistan province in Pakistan.

\section{Setting \\ General setting}

Balochistan is one of the four provinces of Pakistan situated on the southwest part of the country. It is the largest province and covers an area of $347,190 \mathrm{~km}^{2}$, constituting approximately $44 \%$ of the total land area of Pakistan and comprises of 33 districts [25]. In the year 2017, the population estimated at 10.2 million, which scattered across the problematic terrains.

Prison department serves under the provincial home department. There are eleven '11' prisons/jails in Balochistan. Of these, four are the larger "Central" and seven are the relatively smaller "District" jails. Women have no separate jails of their own, and they lodged in a section of men's prison along with their children. Prisons in Balochistan are overcrowded than the actual number of facilities available for intimates, which is one of the key contributing factors to poor prison conditions health 
status [8, 21, 22]. Provision of health services in prisons are under the provincial department of health, includes the provision, management, and development of human resource and regulatory functions. Insufficient and shortage of health staffs in prisons always remain a challenge in Balochistan. Preventive programs such as TB, HIV/AIDS, and hepatitis control programs don't provide permanent screening and management setups within the prisons. Patients presented with symptoms of TB within the hospitals of Jail referred to District Head Quarter (DHQ) hospitals and/or specialized hospital for investigation and diagnoses. Diagnosed patients managed from the facility to follow up visits when needed.

\section{Specific setting}

Central Jail Gaddani is one out of four larger jails in Balochistan prison department. Gaddani is a coastal village situated in tehsil Hub of district Lasbela at the southern part of province Balochistan. This site is both Jail and prison (Jails usually designed to hold inmates awaiting trial or serving a short sentence and prisons designed to hold intimates for long and individuals convicted of more serious crimes) [26]. It serves as the principal imprisonment venue for adult males with illicit drug allegations, as well as for men accused of many other crimes. Central Jail Gaddani is mainly catering the inmates from the districts of Makran and Kalat divisions of Balochistan province. A survey conducted in 2016, indicated a high prevalence of HIV, $(16.6 \%$ in PWIDs, $1.8 \%$ in TGSW and $1.9 \%$ in MSM) in Turbat district Kech and (6.0\% in PWIDs and 3.7\% in MSM) in central Jail Gaddani $[9,10]$, with underlying unprotected sexual, smoking, alcoholism and injecting behaviors, determinants of the TB/HIV co-infection, HBV and HCV infections in prison environment. Rural health center (RHC) Gaddani is the nearest BMU $(5 \mathrm{~km})$, while Jam Ghulam Qadir (JGQ) civil hospital Hub $(25 \mathrm{~km})$ is the nearest Xpert and TB/HIV sentinel site with central Jail Gaddani.

\section{Study population Inclusion criteria}

All prisoners, jail staffs, and staff's family members with the informed consent of central jail Gaddani during the study period included.

\section{Exclusion criteria}

All prisoners, jail staffs, and staff's family members who refused to participate, were not present during screening, seriously ill, known HIV, Hepatitis and tuberculosis patients, excluded from the study.

\section{Sampling methods and sample size}

Central Jail Gaddani consists of 20 barracks of different sizes, in addition to a hospital barrack; prisoner population ranges from 2 to 20 per barrack. We sampled all prisoners, staffs of the Jail, and their family members in the staff's residence colony to obtain more precise estimates of infectious diseases.

\section{Data collection and validation}

Data was collected using a structured data collection form 'An additional file shows this in more detail (see Additional file 1)' by the trained study team. For each prisoner participant included in the study; we determined the status of imprisonment, occupation, drug use, rural/urban residence. The prison ID numbers along with the prisoner's name, sex, and age, which used for tracking the diagnosed patients' on positive/reactive results of screening tests. The study team included trained technical staff for Xpert testing, digital X-ray processing in mobile vans, laboratory techniques (RDTs for HIV and Hepatitis), and sputum sample collection for Xpert testing. A medical officer, chest specialist, and child specialist were available for clinical evaluations and diagnosis. All Jail health care staff and study team were trained/oriented in ethical consideration of privacy and confidentiality.

\section{Outcome definition}

For the study purposes, we defined TB patient if any participant detected MTB by Xpert testing, or clinically diagnosed by study physicians after an abnormal finding on CAD4TB digital X-ray. HIV patients who confirmed on three serial testing RDTs. Hepatitis infected patients who ruled on one serological assay testing reactive result on RDT.

\section{Screening methods \\ Tuberculosis (TB)}

Screening through digital $\mathrm{X}$-ray mobile van linked with CAD4TB software All participants screened through a digital X-ray machine connected with CAD4TB, which can immediately analyze digital images made by digital X-ray machines installed in a mobile van. Digital $\mathrm{X}$-rays can efficiently make large numbers of chest radiographs at a low cost. CAD follows the processing steps, 1Lung shape analysis, 2- Clavicle detection, 3- Texture analysis. Texture within the lung fields and the shape of the extracted lung fields compared with a training database obtained from thousands of training images. Based on this analysis, a grade for the image computed. Based on the category and expected prevalence in the population, the probability that the picture contains signs of TB calculated. The image sent automatically to a separate computer on which the CAD4TB software installed, the program performs the quality check and the image analysis steps, and the result stored on disk. The CAD4TB 
software gives a probability percentage of normal vs. abnormal consistent with TB [27].

.Sputum collection, transportation, and testing through Xpert for MTB/RIF assay Presumptive TB case (PTC) identified by CAD4TB digital X-ray machine and as per National TB guideline, were tested for MTB/ RIF assay by Xpert machine. Sputum samples obtained through front-loading technique (same-day diagnosis) [1]. Samples were preserved in specimen transporting boxes and transported to Xpert site at JGQ hospital hub for processing on the same day.

Diagnosed TB patients were enrolled in treatment for $6 / 8$ months under national TB guideline, with provision of anti TB treatment (ATT) drugs and linked with TB BMU for follow up and completion of treatment.

\section{HIV/AIDS}

Participants with informed consents were screened by rapid diagnostic kits (RDT) according to national HIV testing and counseling (HTC) strategy of three serial testing (Test $1=$ Alere Determine HIV-1/2 Ag/Ab Combo (Sensitivity $>99 \%$ ), Test $2=$ Uni-Gold HIV (Specificity $>99 \%$ ) Test $3=$ SD Bioline HIV-1/2 3.0 (Sensitivity $>99 \%$, Specificity $>98 \%$ ). The CD-4 test for deciding to initiate Anti-Retroviral Therapy (ART) done for participants who found positive on all three following tests (T-1, T-2 \& T-3) [28-30]. ART recommended through ARV centers of the AIDS control program.

\section{Screening for HBV and HCV}

Participants with informed consents screened for HBV and HCV by RDTs and one assay serological testing strategy. Non-reactive participants vaccinated for Hepatitis-B as per the national hepatitis vaccination schedule (1st dose on the spot, 2nd dose after 1 month, and booster dose after 6 months). Participants reactive on initial RDT results were recommended to refer for further HBV DNA and HCV RNA NAT testing to confirm the diagnosis and management at concern point $[29,31,32]$.

\section{Analysis and statistics}

Data were double entered, validated, and analyzed using EpiData software (version 3.1 for entry and 2.2.2.183 for review, EpiData Association, Odense, Denmark). Initially, descriptive statistics were used to describe the study population and diagnosed TB, AIDS, HBV, and $\mathrm{HCV}$ patients.

\section{Results}

A total of 567 participants offered screening, 63\% (356) prisoners, 23\% (129) jail staff's family members, $14 \%$ (82) jail staffs, and less than $1 \%$ refused the screening Fig. 1. Almost all participants were screened initially by questionnaire. The median age of study participants was 30 years (IQR: $21-37$ ) [30 years (IQR: $26 \pm 37$ ) in the prisoners, 32 years (IQR: $24 \pm 39$ ) in the staff's family members and 33 years (IQR: $29 \pm 40$ ) among jail staffs]. Among enrolled majority, participants were male $(85 \%)$. A total of 58 participants detected as $\mathrm{HCV}$ and $\mathrm{HBV}$ reactive on RDT, 24HIV responsive on three series testing, and $05 \mathrm{~TB}$ patients. The essential socio-demographic characteristics summarized in Table 1.

\section{TB diagnostic and CAD4TB probability status}

Among consented; 99.6\% (565/567) participants screened by digital X-ray linked with CAD4TB software for $\mathrm{TB}$, out of $30 \%$ (172) were found with an abnormal suggestion (score $>50$ ) and out of $26 \%$ (148) were tested on Xpert MTB/RIF assay. 2\% 903) of the tested were found MTB detected (Fig. 1). A total of 1\% (05) participants diagnosed as TB patients (02 prisoners, 02 staff's family members, and 01 jail staff). Two children were diagnosed clinically from the staff's family members. The probability of TB diagnosis with CAD4TB suggestion of abnormality (Score $>50$ ) was one time more than in the opinion of normality (Score $<50),(\mathrm{RR}=1.03(95 \% \mathrm{CI}$ : 1.00-1.06) $p=0007)$.

\section{HIV serological testing status}

The HIV seropositivity was $4 \%(24 / 562)$, on three series testing, 24 diagnosed as AIDS patients, and all were prisoners. The detail of three series HIV testing and CD4 count summarized in Table 2. Among 24 HIV reactive participants, 01 dually infected with $\mathrm{TB}$ and 13 with Hepatitis (01 HBV, $12 \mathrm{HCV})$.

\section{Hepatitis serological testing status}

Overall 10\% (58) participants were found hepatitis seropositive; $7.29 \%$ (41) were $\mathrm{HCV}, 2.84 \%$ (16) were $\mathrm{HBV}$, and $0.17 \%$ (01) was both HBV and HCV. Among hepatitis seropositives 49 were prisoners, 08 were jail staffs and 01wasstaff's family member.

\section{Co-morbidities status}

We found co-morbidities among 15 identified infected participants, 01was TB/HIV co-infected, $12 \mathrm{HCV} / \mathrm{HIV}$, $01 \mathrm{HBV} / \mathrm{HIV}$, and $01 \mathrm{HBV} / \mathrm{HCV}$ (Fig. 2). Prisoners were more likely to have multiple infections (100\%) than other participants.

Furthermore; high uptake of infectious diseases detection through new screening tools with minimal utilization of the program resources, human resource and time with a brief orientation to health staffs to utilize these tools for prevention may be particularly feasible, reliable and highly acceptable in prison settings. 


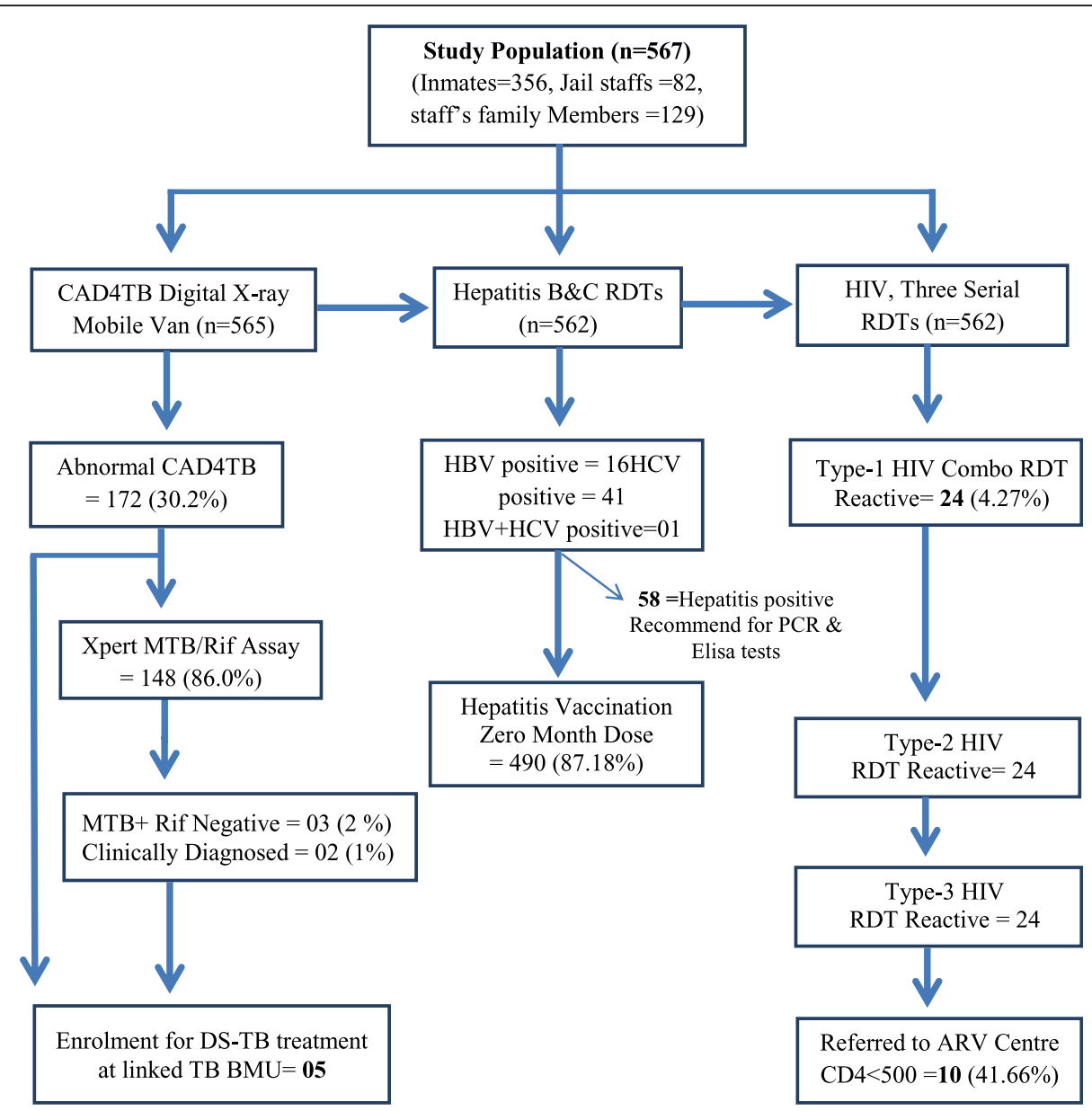

Fig. 1 Flow diagram of systematic screening for HIV, Hepatitis and TB in central Jail Gaddani Balochistan

Table 1 Socio-demographic characteristics of participants screened for HIV, Hepatitis and TB in Central Jail Gaddani, Balochistan

\begin{tabular}{lllll}
\hline & $\begin{array}{l}\text { Jail Inmates } \\
n=356(\%)\end{array}$ & $\begin{array}{l}\text { Jail staffs } \\
n=82(\%)\end{array}$ & $\begin{array}{l}\text { Staff's family } \\
n=129(\%)\end{array}$ & $\begin{array}{l}\text { Total } \\
N=567(\%)\end{array}$ \\
\hline $\begin{array}{lllll}\text { Age in years } \\
\text { 00-14 }\end{array}$ & $0(0.0)$ & $0(0.0)$ & $71(55.0)$ & $71(12.7)$ \\
$15-29$ & $158(44.4)$ & $22(26.8)$ & $29(22.5)$ & $209(36.9)$ \\
$30-44$ & $150(42.1)$ & $49(59.7)$ & $16(12.4)$ & $215(37.8)$ \\
$45-59$ & $29(8.1)$ & $11(13.4)$ & $6(4.7)$ & $46(8.1)$ \\
$60 \&$ above & $19(5.3)$ & $0(0.0)$ & $7(5.4)$ & $26(4.6)$ \\
Sex & & & & \\
Male & $351(98.6)$ & $76(92.7)$ & $57(44.2)$ & $484(85.4)$ \\
Female & $5(1.4)$ & $6(7.5)$ & $72(55.8)$ & $83(14.6)$ \\
Residence & & & & $115(20.3)$ \\
Rural & $78(21.9)$ & $12(20.7)$ & $20(15.5)$ & $452(79.7)$ \\
Urban & $278(78.1)$ & $65(79.3)$ & $109(84.5)$ & \\
\hline
\end{tabular}

HIV Human immunodeficiency virus

\section{Discussion}

Our study determines the high frequency of infectious diseases (HIV, HBV, HCV, and TB) at Central Jail Gaddani, one of the largest prisons in Balochistan. The overall frequency rate of Hepatitis was $9.6 \%$, HIV $4.2 \%$, and TB 1\%. We found the frequency of HIV, Hepatitis, and TB higher than the general population in Pakistan. This result harmonizes with studies conducted in other countries where the frequencies of infectious diseases prevalence among prison inmates were also higher compared to the general population $[2,10,12,14,17]$. The numbers of HIV has found significantly high as compared to the jails of other provinces of the country, which was reported $1-3 \%$ on average [33]. We also noted the comorbidities among the infected participants with higher in HIV-infected individuals. The highest observed of HCV with HIV, because the spread routes of both are common, and so are expected to co-infect the same individuals.

Detection of significant infectious diseases patient's in jail staffs and their family members along with intimates evident the consequences of infectious diseases in 
Table 2 Screening \& diagnostic tests results for HIV, Hepatitis and TB in central Jail Gaddani, Balochistan

\begin{tabular}{|c|c|c|c|c|}
\hline \multirow[t]{2}{*}{ Infection \& Tools } & Jail Inmates & Jail staffs & Staff's family & Total \\
\hline & $n=356(\%)$ & $n=82(\%)$ & $n=129(\%)$ & $N=567(\%)$ \\
\hline \multicolumn{5}{|l|}{ Tuberculosis (TB) } \\
\hline CAD4TB digital X-ray & $356(100)$ & $82(100)$ & $127(98.4)$ & 565 (99.6) \\
\hline Abnormal (Score > 50) & $109(30.6)$ & $26(31.7)$ & $37(28.7)$ & $172(30.4)$ \\
\hline X-pert MTB/Rif Assay & $120(33.7)$ & 12 (14.6) & $16(12.4)$ & $148(26.1)$ \\
\hline MTB+ Rif-^^ & $02(0.6)$ & $01(1.2)$ & $00(0.0)$ & $03(0.5)$ \\
\hline Clinically diagnosed & $00(0.0)$ & $00(0.0)$ & $02(100)$ & $02(0.4)$ \\
\hline HIV Three Serial RDTs & $356(100)$ & $81(98.8)$ & $125(96.9)$ & $562(99.1)$ \\
\hline Type-1 reactive & $24(6.7)$ & $00(0.0)$ & $00(0.0)$ & $24(4.3)$ \\
\hline Type-2 reactive & $24(6.7)$ & $00(0.0)$ & $00(0.0)$ & $24(4.3)$ \\
\hline Type-3 reactive & $24(6.7)$ & $00(0.0)$ & $00(0.0)$ & $24(4.3)$ \\
\hline CD4 Count ${ }^{a}$ & $19(79.1)$ & $00(0.0)$ & $00(0.0)$ & $19(79.1)$ \\
\hline$<500$ & $10(2.8)$ & $00(0.0)$ & $00(0.0)$ & $10(1.77)$ \\
\hline Hepatitis Positive RDT & $356(100)$ & $82(100)$ & $122(94.6)$ & $560(98.8)$ \\
\hline Hepatitis B positive & $15(4.2)$ & $01(1.2)$ & $00(0.0)$ & $16(2.9)$ \\
\hline Hepatitis C positive & $37(10.4)$ & $03(3.7)$ & $01(0.8)$ & $41(7.3)$ \\
\hline HBV+ HCV positive & $01(0.3)$ & $00(0.0)$ & $00(0.0)$ & $01(0.2)$ \\
\hline
\end{tabular}

a CD4 Count done for reactive on 3 series HIV RDTs; CAD4TB Computer aided detection for TB, RDTs Rapid diagnostic tests, MTB+/Rif- Mycobacterium tuberculosis Detected/Rifampicin resistant not detected, HIV Human immunodeficiency virus, HBV Hepatitis B Virus, HCV Hepatitis C Virus

prisons which are not restricted to within the prisons. These contagious infections from inmates possibly will spread through visitors, prison staff, and released inmates into the community, thereby make vulnerable the efforts done in the general population for the control of infectious diseases.

In this study, active case finding with newly recommended sensitive and specific screening tools for infectious diseases detected 58 cases of Hepatitis, 24 cases of HIV, and 05 cases of TB at Central Jail Gaddani. Screening by digital X-ray linked with CAD4TB evident the time and actively diagnosis of TB patients with its suggestion $($ Score $<50)$ of abnormality, which was one time more than the normal. The outcome of this study, therefore, highlights the significance of active case finding of infectious diseases in prisons. The choice of the best latest recommended screening tools and diagnostic algorithms will remain an essential deliberation in the control of contagious diseases in prisons.

There were several strengths of the study. First, the study had a representative sample size and screened all the prisoners, jail staffs, and staff's family members with

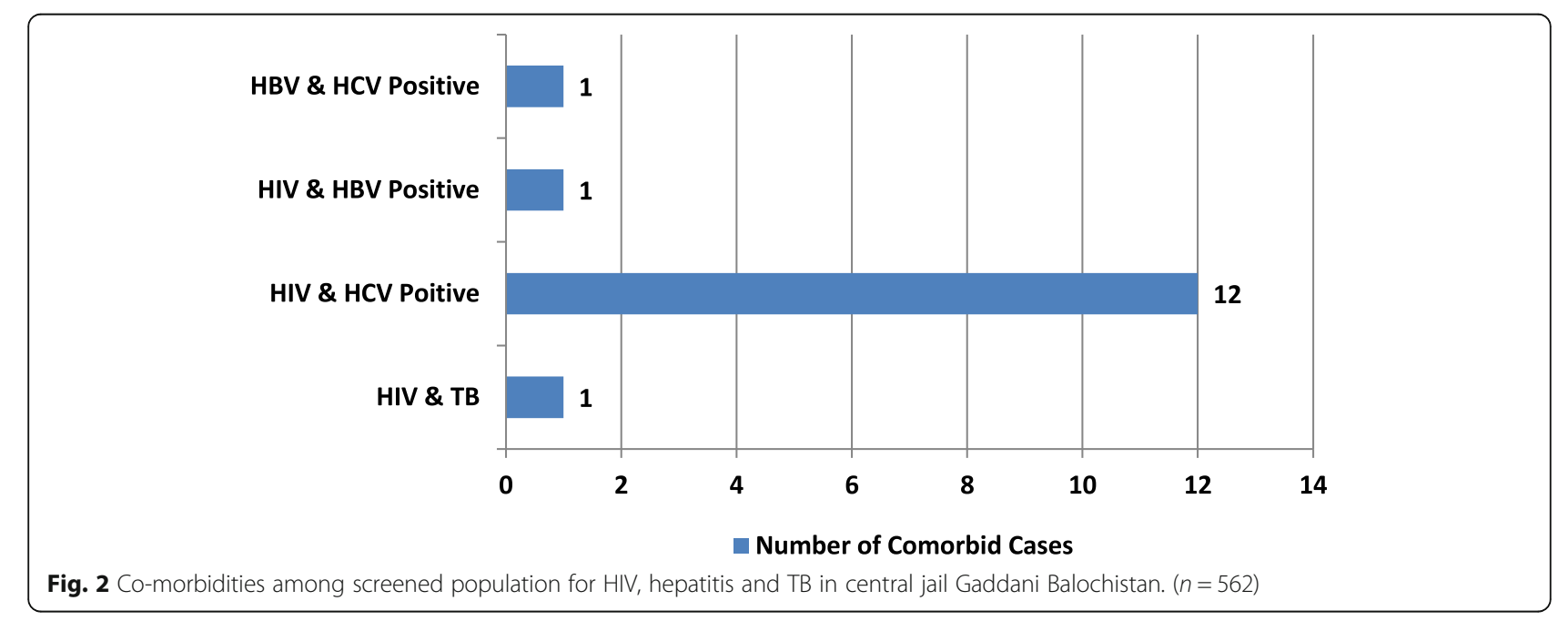


a high response rate. Second, our action plan provided better logistic measures to systematically use latest screening tools and diagnostic algorithms with high sensitivity and specificity for all three major infectious diseases (digital X-ray linked with CAD4TB software, sputum testing on Xpert, WHO recommended three series RDTs for HIV, and Hepatitis, and CD4 count for HIV reactive patients). Each of these tests considered separately have their limitations, and taken together; they provide us with a higher assured estimate that should be regarded with caution. All the procedures support us for robust convinced estimates. Third, we also used an arrangement of clinical criteria with acid-fast bacilli (AFB) and radiological screening to document non-culture proven TB. Fourth, the study used the resources of the existing program in collaboration with other programs. Fifth, all participants found eligible for Hepatitis-B, vaccination was vaccinated and linked with current district hepatitis vaccination program for completion of the remaining doses as per schedule.

Limitations of this study include our incapacity to assess incidence due to cross-sectional study design, research of a particular jail due to budget constraints, and we had insufficient numbers of actions to examine risk factors for infectious diseases. Finances limited the extent of our workup and the number of jails inclusion for infections that we could diagnose.

Based on the study results, we presumed the possible reasons for the remarkably high frequency of infectious diseases in prisons, could be explained by; One, the conditions of the deprived detention. Second, inadequate health care services because the prison health system works in complete isolation from the overall healthcare system. Third, most often underfunded prison's health care system. Fourth, the lack of screening facilities from infectious diseases controls programs: fifth, lack of awareness and counseling of the detected patients with contagious diseases. Sixth, the newly admitted prisoners, do not screen for infectious disease at the time of the first admission.

There are several program implications and considerable efforts that can be done to address the challenge of the high prevalence of infectious diseases in the prisons, which outlined in the study discussion. First, it will be necessary to take immediate actions for the betterment of prisons conditions and decrease overcrowding. Formulation of strategies based on the recommendations of different commissions and committees to reform the prison system in Pakistan is urgently needed [25]. Second, the prison healthcare system needs for increased funding and should recognize as an integral part of the public health sector. It shall advance from its present reactive "sick call" model into a proactive system that emphasizes early disease detection and treatment, health promotion, and disease prevention. Third, prison healthcare services should directly work with national infectious disease control programs to make sure that prison inmates are regularly screened for infectious diseases. Those detected positive should be put on treatment without delay by linking them with the nearest infectious disease controlling health care facility. Finally, all prisoners must be screened for infectious diseases prior to admittance into the prison/jails, and any prisoner with an infectious disease should be properly tracked up after release from the prison/jail to ensure completion of a particular treatment and/or vaccination [34].

\section{Conclusion}

The study advocates high frequency of infectious diseases among prisoners in prisons of Balochistan, Pakistan. To limit the transmission of infections, it is necessary to take steps for improvement of prisons conditions and application of recommended screening protocols at the time of the first entry of prisoners in the prisons.

\section{Supplementary information}

Supplementary information accompanies this paper at https://doi.org/10. 1186/s12889-019-8011-7.

Additional file 1. Data Collection Form being utilized during data collection process.

\section{Abbreviations}

AFB: Acid-fast bacilli; AIDS: Acquired Immunodeficiency Deficiency Syndrome; ART: Anti Retro Viral Treatment; ARV: Anti Retro Viral; ATT: Anti TB treatment; BMU: Basic Management Unit; CAD4TB: Computer-Aided Detection for TB; HBV: Hepatitis-B Virus; HCV: Hepatitis-C Virus; HIV: Human Immunodeficiency Virus; IG: Inspector General; JGQ: Jam Ghulam Qadir; MTB+: Mycobacterium tuberculosis Positive; RDTs: Rapid Diagnostic Tools; RHC: Rural health center; TB: Tuberculosis

\section{Acknowledgments}

This study conducted which resulted in this publication through the collaborative partnerships of provincial TB control program Balochistan (PTP), national TB control program Pakistan (NTP), provincial AIDS control program Balochistan (PACP), provincial Hepatitis control program Balochistan, provincial prisons and home department government of Balochistan Quetta, The Indus Hospital Karachi $(\mathrm{TIH})$, Bridge Consultant Foundation and Strengthening Participatory Organization (SPO).

\section{Authors' contributions}

Conceptualization: AW, NS, Designing of the work: AW, NS, DK, SA, Acquisition: AW, HR, BA, AQ, Analysis of data: AW, AY, RF, Interpretation of data and results: AW, NS, RF, AY, DK, BA, HR, Creation of data collection form used in the work: AW, BA, DK, Drafted and revised the work: AW, NS, ZS, AY, $R F, A Q, S K$, All authors read and approved the final manuscript.

\section{Funding}

No specific funding was available or obtained from any organization for this study. It conducted through a collaborative venture within the available program's resource. Implementing expanses of the study team, Xpert cartages, Type-1 Combo HIV kits, ATT drugs, Tab INH for prevention for HIV positive patients and logistics were supported by provincial TB control program Balochistan. A mobile van equipped with digital X-ray linked with CAD4TB software with reading the cost of the software, HR, and logistics required for van utilization was provided by partner "The Indus Hospital Karachi." Type-2 and Type-3 HIV RDT kits, portable CD4 machine were provided 
by provincial AIDS control program Balochistan. Hepatitis RDT kits and hepatitis vaccines were provided by provincial Hepatitis control programs Balochistan. Trained counselors for HIV awareness were provided by Bridge Consultant Foundation. Publication fee in an international journal was not available from any of the organization.

\section{Availability of data and materials}

The datasets generated and/or analyzed during the current study are not publicly available due to maintaining the confidentiality of participants keeping in view the ethical consideration for stigmatized infectious diseases i.e. TB and HIV but are available from the corresponding author on reasonable request.

\section{Ethics approval and consent to participate}

Ethics approval obtained from the Ethical Review Committee of Bridge Consultant Foundation, Pakistan. Written consent was obtained from al screened participants on a developed data collection form. It had explained to all participants that their acceptance or refusal to take part in the survey would not affect their imprisonment. Their possibility for parole in case of prisoners, and not benefited to the jobs in case of jail staffs.

\section{Consent for publication}

Not applicable.

\section{Competing interests}

The authors declare that they have no competing interests.

\section{Author details}

'Provincial Tuberculosis Control Program, Health Department Government of Balochistan, Building Western Bypass Link Brewery Road, Quetta, Balochistan, Pakistan. ${ }^{2}$ National Tuberculosis Control Program, Ministry of NHSR\&C, Islamabad, Pakistan. ${ }^{3}$ Provincial AIDS Control Program, Quetta, Balochistan, Pakistan. ${ }^{4}$ Social and Health Inequalities Network Pakistan, Quetta, Pakistan. ${ }^{5}$ University of Bergen, Bergen, Norway. ${ }^{6}$ Mercy Corps, Quetta, Pakistan.

${ }^{7}$ Faculty of Bolan Medical University, Quetta, Pakistan.

Received: 3 July 2019 Accepted: 27 November 2019

Published online: 04 December 2019

\section{References}

1. Ministry of Health Pakistan. Pakistan TB Guidelines. 2015.

2. Chimphambano C, Komolafe I, Muula A. Prevalence of HIV, HBsAg and Hep C antibodies among inmates in Chichiri prison, Blantyre, Malawi. Malawi Med J. 2007;19(3):107-10.

3. Kazi A, Shah S, Jenkins C. Risk factors and prevalence of tuberculosis, human immunodeficiency virus, syphilis, hepatitis B virus, and hepatitis $C$ virus among prisoners in Pakistan. Int J Infect Dis. 2010;14:1-15.

4. Gurwood AS, Kabat AG. World Prison Population List, Eleventh Edition. Institute for Criminal Policy Research (ICPR) 2009. p. 1-15.

5. Gorar ZA, Zulfikar I. Seropositivity of hepatitis $C$ in prison inmates of Pakistan--a cross-sectional study in prisons of Sindh. J Pak Med Assoc. 2010; 60(6):476-9.

6. Kamarulzaman A, Reid SE, Schwitters A, Wiessing L, El-Bassel N, Dolan K, et al. Prevention of transmission of HIV, hepatitis B virus, hepatitis $C$ virus, and tuberculosis in prisoners. Lancet. 2016;388(10049):1115-26.

7. van den Bergh BJ, Gatherer A, Fraser A, Moller L. Imprisonment and women's health: concerns about gender sensitivity, human rights, and public health. Bull World Health Organ. 2011;89(9):689-94.

8. Baloch SU. Prisons in Balochistan: rehabilitation centers or cages for brutes women have no separate jails of their own in balochistan, and they are lodged in a section of men' $s ; 2016$.

9. Behavioural I, Surveillance B, Populations FK. Government of Balochistan Balochistan AIDS control program health Department for key Populations, In selected cities of Balochistan submitted by; 2016.

10. Hiv G, In S. Second generation HIV surveillance in Pakistan round 5 integrated biological \& behavioral surveillance in Pakistan; 2016.

11. Macalino GE, Vlahov D, Sanford-Colby S, Patel S, Sabin K, Salas C, et al. Prevalence and incidence of HIV, hepatitis B virus, and hepatitis $C$ virus infections among males in Rhode Island prisons. Am J Public Health. 2004; 94(7):1218-23.
12. WHO Regional Office for Europe. Good governance for prison health in the 21st century; 2013. p. 1-9. -19

13. Sultan F, Khan A. Infectious diseases in Pakistan: a clear and present danger. Lancet. 2013;381(9884):2138-40.

14. Global tuberculosis report 2017. Geneva: World Health Organization; 2017. Licence: CC BY-NCSA 3.0 IGO.

15. Pervaiz A, Sipra FS, Rana TH, Qadeer I. Pre-donation screening of volunteer prisoner blood donors for hepatitis B and C in prisons of Punjab, Pakistan. J Ayub Med Coll Abbottabad. 2015;27(4):794-7.

16. Global tuberculosis report 2018. Geneva: World Health Organization; 2018. Licence: CC BY-NC-SA 3.0 IGO.

17. Estimates A. Country factsheets Pakistan | 2017 HIV and AIDS estimate adults and children living with country factsheets Pakistan | 2017 HIV testing and treatment cascade People living with HIV Coverage of adults and children; 2017. p. 1-7.

18. World Health Organization. Global hepatitis report, 2017; 2017. p. 62.

19. Dara M, Acosta CD, Melchers NVSV, Al-Darraji HAA, Chorgoliani D, Reyes H, et al. Tuberculosis control in prisons: current situation and research gaps. Int J Infect Dis. 2015;32:111-7.

20. The S, To D, Wealth P. Provincial Strategic Plan. 2010.

21. United States Department of Labour. Pakistan 2016 Human Rights Report. 2016.

22. Achakzai J, Bukhari S, Quetta DJ. Jahangir Achakzai \& Shakira Bukhari Situation and Needs Assessment of Women in Jails A Case Study of Province of Balochistan; 1957. p. 38-43.

23. Qureshi H, Bile KM, Jooma R, Alam SE, Afridi HUR. Prevalence of hepatitis B and $C$ viral infections in Pakistan: findings of a national survey appealing for effective prevention and control measures. EMHJ. 2010; vol 16

24. Dolan K, Larney S. HIV in Indian prisons: Risk behaviour, prevalence, prevention \& treatment. 2010:696-700.

25. Akbar MS, Hussain BM. Prison reforms and situation of prisons in Pakistan; 2009.

26. MacGill M. What is the difference between, vol. 1984; 1994. p. 30754.

27. World Health Organization. Netherlands: Medical devices and eHealth solutions, Computer-aided detection for Tuberculosis 2011 - 2012.

28. Manabe YC, Wang Y, Elbireer A, Auerbach B, Castelnuovo B. Evaluation of portable point-of-care CD4 counter with high sensitivity for detecting patients eligible for antiretroviral therapy. PLoS One. 2012;7(4):1-5.

29. World Health Organization. HIV assays operational characteristics. Geneva: HIV rapid diagnostic tests (detection of HIV-1/2 antibodies); report 17. 2013

30. Test RA. Year prequalified Type of assay Product Name Product code(s) Regulatory version Manufacturer Manufacturing site(s) Packaging 2015 CD4 Technologies Aquios CL flow cytometer; 2016.

31. Organization WH. Guidelines on Hepatitis B and C Testing; 2017. p. 1-170.

32. Bosan A, Qureshi H, Bile KM, Ahmad I, Hafiz R. A review of hepatitis viral infections in Pakistan. J Pak Med Assoc. 2010;60(12):1045-58.

33. Aziz S, Wali A, Fatima R, Yaqoob A. Prevalence and associated risk factors of HIV in prisons in Balochistan, Pakistan: a cross-sectional study. Muhammad Dawood Khan. 2018;0:1-9.

34. Ekundayo EO, Onuka O, Mustapha G, Geoffrey M. Active case finding of pulmonary tuberculosis among prison inmates in aba Federal prison, Abia state, Nigeria. Adv Infect Dis. 2015;5(1):57-62.

\section{Publisher's Note}

Springer Nature remains neutral with regard to jurisdictional claims in published maps and institutional affiliations.

\section{Ready to submit your research? Choose BMC and benefit from:}

- fast, convenient online submission

- thorough peer review by experienced researchers in your field

- rapid publication on acceptance

- support for research data, including large and complex data types

- gold Open Access which fosters wider collaboration and increased citations

- maximum visibility for your research: over $100 \mathrm{M}$ website views per year

At $\mathrm{BMC}$, research is always in progress.

Learn more biomedcentral.com/submission 